\title{
Farnesol, a Fungal Quorum-Sensing Molecule Triggers Candida Albicans Morphological Changes by Downregulating the Expression of Different Secreted Aspartyl Proteinase Genes
}

\author{
Nadège Décanis ${ }^{1}$, Neftaha Tazi ${ }^{1}$, Alexandra Correia ${ }^{2}$, Manuel Vilanova ${ }^{3,4}$ and Mahmoud Rouabhia ${ }^{1, *}$ \\ ${ }^{I}$ Groupe de Recherche en Écologie Buccale, Faculté de Médecine Dentaire, Université Laval, Québec, Québec, GlV \\ OA6, Canada \\ ${ }^{2}$ Centro de Biologia Molecular e Ambiental (CMBA), Departamento de Biologia, Universidade do Minho, Campus de \\ Gualtar, Braga, Portugal \\ ${ }^{3}$ Laboratório de Imunologia Mário Arala Chaves, ICBAS - Instituto de Ciências Biomédicas de Abel Salazar- \\ Universidade do Porto, Porto, Portugal \\ ${ }^{4}$ IBMC - Instituto de Biologia Celular e Molecular, Porto, Portugal
}

\begin{abstract}
The aim of this study was to determine the effect of exogenous farnesol in yeast-to-hyphae morphogenesis, and Saps $(2,4,5$ and 6) mRNA expressions by a Candida strain that does not produce endogenous farnesol. $C$. albicans was cultured in the absence and presence of farnesol at various concentrations $(10,100$, and $300 \mu \mathrm{M})$, in proteinase induction medium, and then used to determine yeast-to- hyphae changes, Candida ultrastructure and to determine Saps 2, 4, 5 and 6 expressions using q-TR-PCR and ELISA (for Sap2). Data demonstrated that farnesol greatly reduced the yeast-to-hyphae morphogenesis of a Candida strain that does not produce endogenous farnesol. Farnesol induced several ultrastructural alterations, including changes in the cell-wall shape, a visible disconnection between the cell wall and cytoplasm with an electron-lucent zone between them, and the presence of electron-dense vacuoles. Tested on gene expressions, farnesol was able to significantly $(\mathrm{p}<0.01)$ decrease Sap2 secretion and mRNA expression. Farnesol downregulated also Sap4-6 mRNA expression. These results demonstrated for the first time that farnesol modules Candida morphogenesis through a downregulation of Saps 2, 4, 5 and 6 expressions. Overall these data point to the potential use of farnesol as an antifungal molecule.
\end{abstract}

Keywords: Candida, farnesol, transition, saps.

\section{INTRODUCTION}

Candida is a fungus, which accounts for nearly $96 \%$ of all opportunistic mycoses and is the fourth most common bloodstream pathogen in North American and seventh in European hospitals [1, 2]. Worldwide, candidiasis is associated with overall severity of illness, increased rate of invasive interventions, use of broad spectrum antibiotics and parenteral nutrition, which are all unavoidable among critically ill patients $[3,4]$. Candida infections lead to a prolonged stays in intensive care unit and hospital which causes significant health and economic problems [5, 6]. Among the Candida genus, Candida albicans is a fungal organism that forms part of the normal oral microbial flora in approximately $50 \%$ of healthy individuals [7]. C. albicans is a polymorphic organism and can grow as yeast, pseudohyphae or hyphae; each differing in its morphology and virulence [8]. The yeast form is commonly associated with the commensal

\footnotetext{
*Address correspondence to this author at the Faculté de Médecine Dentaire, Pavillon de Médecine Dentaire, Local 1728, Université Laval, 2420 rue de la Terrasse, Québec, Québec, Canada G1V 0A6, Ext. 16321; Tel: (418) 656-2131; Fax: (418) 656-2861;

E-mail: Mahmoud.Rouabhia@fmd.ulaval.ca
}

carrier state, thanks to the innate immune function. The disruption of the immune surveillance leads to $C$. albicans growth and morphological changes to its hyphal form facilitating tissue invasion and pathogenesis $[9,10]$. C. albicans proceeds in different ways to invade and infect its host. These include its ability to overcome the host immune response [11], the phenotypic transition from blastospore to hyphal form [12], the use of hyphae-associated factors [13], invasion-like molecules [14], and secreted aspartyl proteinases (Saps) [15].

Saps constitute a family of 10 proteolytic enzymes known as secreted aspartyl proteinases [16]. The contribution of Saps to mucosal and systemic infections and their involvement in adherence, tissue damage, and evasion of host immune responses has been reported [17]. Sap2 is essential to $C$. albicans growth in protein-containing media [17]. Sap1 and Sap3 are expressed during phenotypic switching $[18,19]$, while Sap4, Sap5 and Sap6 are expressed upon hyphal formation [20]. Sap1 to Sap6 as well as Sap9 and Sap10 are involved in the adhesion mechanism to host cells [21]. Although these 10 Sap proteins are produced during infection, their induction time points and expression levels occur at different stages. When in contact with host tissue 
and cells, C. albicans is capable of growing as yeast, pseudohyphae, and hyphae. Candida density is one of several factors that influence hyphal growth [22]. Some studies have revealed that the population dependence of $C$. albicans morphology results from the continuous production and detection of sesquiterpene farnesol (3, 7, 11-trimethyl-2, 6, 10dodecatriene-1-ol) [23].

Farnesol is a metabolic product of mevalonate/sterol synthesis in eukaryotes [24]. Farnesol generally functions endogenously; however, in C. albicans, this molecule acts in an autocrine manner. Research has shown that farnesol produced in situ by planktonic $C$. albicans cultures prevented biofilm formation [25]. The accumulation of farnesol blocks the morphological shift from yeast to hyphae form at high cell densities [26-29]. These data suggest that farnesol may reduce Candida pathogenesis through a downregulation of yeast-to hypha morphogenesis that could involve a modulation of Saps gene expression. The purpose of this study was to investigate the effect of exogenous farnesol on yeast-tohypha morphogenesis, Candida ultrastructural shape and Saps gene expression.

\section{MATERIALS AND METHODS}

\section{Farnesol Solution}

The trans, trans-farnesol was purchased from SigmaAldrich Canada Ltd. (Oakville, ON, Canada). Farnesol (1 M) stock was prepared fresh in methanol prior to each experiment. The original flask containing farnesol was de-gassed with nitrogen gas after each use to prevent farnesol degradation by oxygen. In this study, farnesol was used at 10, 100 and $300 \mu \mathrm{M}$, These concentrations were comparable to those previously used in different studies [26].

\section{Candida Strain}

We selected $C$. albicans (ATCC 10231) which is known to be a nonproducer of endogenous farnesol [30]. This allows us to have specific effect, if any, related to exogenous farnesol. C. albicans was cultured for $24 \mathrm{~h}$ on Sabouraud dextrose agar plates (Becton Dickinson, Oakville, ON, Canada) at $30^{\circ} \mathrm{C}$. For the $C$. albicans suspensions, one colony was used to inoculate $10 \mathrm{ml}$ of Sabouraud liquid medium supplemented with $0.1 \%$ glucose, $\mathrm{pH} 5.6$. The cultures were grown to the stationary phase in a shaking water bath for $18 \mathrm{~h}$ at $30^{\circ} \mathrm{C}$. The yeast cells were then collected, washed with phosphate buffered saline (PBS), counted by means of a haemocytometer and adjusted to $10 \% \mathrm{ml}$ prior to use.

\section{Effect of Farnesol on C. Albicans Transition}

To determine the effect of farnesol on the yeast-tohyphae transition, C. albicans $\left(10^{4}\right.$ cells) was grown in $10 \mathrm{ml}$ of proteinase induction medium (PIM: $2 \%$ glucose, $0.1 \%$ $\mathrm{KH} 2 \mathrm{PO} 4,0.05 \% \mathrm{MgSO} 4$; adjusted to $\mathrm{pH} 4.0$ ) supplemented with $10 \%(\mathrm{v} / \mathrm{v})$ bovine serum albumin (BSA, Sigma) as previously reported [31] for $24 \mathrm{~h}$ at $37^{\circ} \mathrm{C}$. Farnesol was added with and without fetal bovine serum (FBS) at various concentrations $(10,100$, and $300 \mu \mathrm{M})$. The cultures were incubated at $37^{\circ} \mathrm{C}$, observed microscopically at 6 and $24 \mathrm{~h}$, and photographed to record $C$. albicans morphology. To calculate the percentage of yeast cells that underwent the morphological transition, three aliquots from each culture $(n=5)$ were used to determine the number of yeast cells and hyphae by means of a haemocytometer and an optical microscope, as previously described [32]. The percentage of cells that underwent morphological transition was determined using the following formula: (number of hyphae divided by the number of yeast cells and hyphae $) \times 100$.

\section{Effect of Farnesol on C. Albicans Ultrastructure}

C. albicans $\left(10^{6}\right.$ cells) was cultured in Sabouraud liquid medium in the presence of farnesol at 10, 100, and $300 \mu \mathrm{M}$. C. albicans cultured in the presence of $0.1 \%$ methanol and in the presence of $5 \mu \mathrm{g} / \mathrm{ml}$ of amphotericin-B (Amph-B) constituted the negative and positive controls, respectively. Following a 24-h culture, pellets of $C$. albicans were prepared and used for scanning and transmission electron microscopy analyses.

\section{Scanning Electron Microscopy}

C. albicans cells were fixed in $3 \%(\mathrm{v} / \mathrm{v})$ gluteraldehyde in PBS ( $\mathrm{pH} \mathrm{7.4)} \mathrm{and} \mathrm{dehydrated} \mathrm{in} \mathrm{increasing} \mathrm{concentrations}$ of ethanol $(10 \%, \mathrm{v} / \mathrm{v}$, increments to $100 \%)$. A drop was placed onto a round glass cover slip for critical-point drying (CPD 030; Balzers, Liechtenstein, Germany). The sample was mounted onto an aluminium slab using silver paint (Agar Scientific, Stansted, UK). Sputter coating was performed (S150B, Edwards, London, UK) and the image was viewed using the scanning electron microscope model Jeol JSM 6360 LV (Soquelec, Montréal, QC, Canada). The experiment was repeated three times.

\section{Transmission Electron Microscopy}

C. albicans was fixed in cacodylate buffer, $\mathrm{pH} 6.9$, containing $1 \%(\mathrm{v} / \mathrm{v})$ paraformaldehyde and $2 \%(\mathrm{v} / \mathrm{v})$ gluteraldehyde, for $1 \mathrm{~h}$ at $4{ }^{\circ} \mathrm{C}$. The suspensions were then centrifuged and dehydrated in successive washes of ethanol for $30 \mathrm{~min}$. The $C$. albicans pellets were embedded in Spurr resin and ultra-thin sections were obtained (Ultratome III, LKB, Bromma, Sweden). These sections were then mounted onto a $0.5 \%$ Pioloform (in chloroform)-coated $3.05 \mathrm{~mm}$ copper grid and stained with $3 \%$ aqueous uranyl acetate and $0.1 \%$ lead citrate prior to imaging under a JEOL 1200 transmission electron microscope (Soquelec, Montréal, QC, Canada). The experiment was repeated four times.

\section{RT-PCR Analyses to Assess the Effect of Farnesol on $C$. Albicans Gene Expression}

\section{Total RNA Extraction}

C. albicans $\left(10^{5}\right.$ cells) was cultured in the absence and presence of farnesol at various concentrations (10, 100, and $300 \mu \mathrm{M})$ in PIM supplemented with $10 \%(\mathrm{v} / \mathrm{v})$ bovine serum albumin for $24 \mathrm{~h}$ at $37^{\circ} \mathrm{C}$. Cultures containing $2 \times 10^{7} \mathrm{Can}$ dida were then centrifuged at $110 \mathrm{~g}$ for $10 \mathrm{~min}$, pellets were washed and suspended in $400 \mu \mathrm{l}$ of TES (10 mM Tris-Cl, $\mathrm{pH}$ 7.5; $10 \mathrm{mM}$ EDTA and $0.5 \%$ SDS) and $400 \mu \mathrm{l}$ of phenol acid [33]. The suspension was vortexed for $10 \mathrm{~s}$ and incubated for $45 \mathrm{~min}$ at $65^{\circ} \mathrm{C}$ in a water bath. The suspension was then centrifuged using a microcentrifuge for $5 \mathrm{~min}$ at top speed at $4^{\circ} \mathrm{C}$, after which time the aqueous phase was collected and $400 \mu \mathrm{l}$ of phenol acid were added. The mixture was incubated for $5 \mathrm{~min}$ at $4^{\circ} \mathrm{C}$ and then centrifuged in a microcentri- 
fuge for $5 \mathrm{~min}$ at top speed. The aqueous phase was collected, supplemented with $400 \mu 1$ of chloroform, and incubated for $5 \mathrm{~min}$. Following top-speed centrifugation for $5 \mathrm{~min}$ at $4^{\circ} \mathrm{C}$, the aqueous phase was collected and total RNA was precipitated using ethanol plus $3.5 \mathrm{M}$ sodium acetate, $\mathrm{pH}$ 5.3. Each RNA pellet was re-suspended in $1 \mathrm{ml}$ of ethanol and centrifuged. The pellets were then re-suspended in a low volume of RNAse-free water. RNA concentration was determined by fluorescence using Ribogreen (Molecular Probes Inc., Eugene, OR, USA).

\section{Quantitative Real-Time PCR (qRT-PCR)}

RNA ( $1 \mu \mathrm{g}$ of each sample) was reverse transcripted into cDNA using Maloney murine leukemia virus (M-MLV) reverse transcriptase (Invitrogen Life Technologies, Mississauga, ON, Canada) and random hexamers (Amersham Pharmacia Biotech, Inc., Baie d'Urfé, QC, Canada). RT conditions were $10 \mathrm{~min}$ at $65^{\circ} \mathrm{C}, 1 \mathrm{~h}$ at $37^{\circ} \mathrm{C}$, and $10 \mathrm{~min}$ at $65^{\circ} \mathrm{C}$. Quantitative PCR was carried out as described $[33,34]$. Amounts of mRNA transcripts were measured using the Bio-Rad CFX96 real-time PCR detection system (Bio-Rad, Mississauga, ON, Canada).

Reactions were performed using a PCR supermix from Bio-Rad (iQ SYBR Green supermix). Primers Table 1 were added to the reaction mix at a final concentration of $250 \mathrm{nM}$. Five microliters of each cDNA sample were added to a $20 \mu \mathrm{l}$ PCR mixture containing $12.5 \mu 1$ of iQ SYBR Green supermix (Bio-Rad) and $0.5 \mu 1$ of specific primers (Sap2, Sap4, Sap5, Sap6, and ACT-1) (Medicorp, Inc., Montréal, QC, Canada) and $7 \mu \mathrm{l}$ of Rnase- and Dnase-free water (MP Biomedicals, Solon, OH, USA). Each reaction was performed in a Bio-Rad MyCycler ${ }^{\mathrm{TM}}$ Thermal Cycler (Bio-Rad). For the qPCR, the CT was automatically determined using the accompanying Bio-Rad CFX manager. Thermocycling conditions for the Sap quantitation were $15 \mathrm{~min}$ at $95^{\circ} \mathrm{C}$, followed by 45 cycles for $15 \mathrm{sec}$ at $94^{\circ} \mathrm{C}$ and $1 \mathrm{~min}$ at $60^{\circ} \mathrm{C}$, then $30 \mathrm{~s}$ at $72^{\circ} \mathrm{C}$, with each reaction done in triplicate. The specificity of each primer pair was verified by the presence of a single melting temperature peak. ACT-1 produced uniform expression levels varying by less than $0.5 \mathrm{CT}$ s between sample conditions and was therefore used as a reference gene for this study.

\section{Induction of Sap Protein Production}

To determine farnesol's effect on C. albicans Sap production, $100 \mathrm{ml}$ of a $10^{7}$ yeast cell suspension were grown in the presence or absence of farnesol in proteinase induction medium PIM supplemented with $10 \%$ bovine serum albumin. Candida cultures in the absence of farnesol and presence of pepstatine-A at $50 \mu \mathrm{g} / \mathrm{ml}$ were used as negative (no Sap activity) controls. Candida cultures without farnesol and with PIM that contains or not methanol served as positive controls, whereas the cultures without farnesol and BSA were used as internal negative controls (no Sap production). Each concentration was simultaneously tested in triplicate (in separate tubes). These were incubated for 8 days at $37^{\circ} \mathrm{C}$ under agitation. Farnesol concentrations were refreshed every $24 \mathrm{~h}$. This relatively long experimental duration time has previously been shown to be required for sufficient Sap production [31]. On the last day of culture, the supernatants were collected, centrifuged at $1200 \mathrm{~g}$ for $10 \mathrm{~min}$, and used to determine Sap2 concentration in each sample by ELISA.

\section{Detection of Sap2 Protein in the Candida Supernatants: Competitive Binding Inhibition ELISA}

Anti-Sap2 IgG antibody was obtained by immunizing male Balbc/c mice with recombinant Saps2 protein [35]. Sera of immunized and sham-immunized mice were collected after 21 days and used to purify anti-Sap2 IgG antibody. Following purification, the reactivity of the anti-Sap2 IgG antibody was determined by ELISA using recombinant Sap proteins rSap1, rSap2, rSap3, rSap5, rSap6 (a kind gift from Dr. Bernard Hube, Hans Knoell Institute, Jena, Germany) and native Sap2 (Brenntag, Denmark). This showed that our anti-Sap2 IgG reacted with both the recombinant and native Sap2 and, to a lesser extent, Sap1 and Sap3. AntiSap2 IgG did not react with other Saps. Anti-Sap2 IgG antibody was thus used to measure the Sap 2 production by $C$. albicans following treatment with farnesol. Polystyrene microtitre plates (Nunc) were coated with $50 \mu \mathrm{l}$ of $5 \mu \mathrm{g} / \mathrm{ml}$ rSap2 in $0.06 \mathrm{M}$ sodium carbonate buffer ( $\mathrm{pH} 9.6)$ and incubated at $4{ }^{\circ} \mathrm{C}$. The wells were then saturated for $2 \mathrm{~h}$ at room temperature with $2 \%$ BSA in Tris-Buffered Saline and Tween 20 (TBST). The plates were washed three times with TBST, after which time $100 \mu$ of each incubation mixture

Table 1. Description of Oligoncleotide Primer Pairs Used in PCR Reactions

\begin{tabular}{|c|l|c|}
\hline Gene & Primer Sequence (5' to 3') & Amp Size (bp) \\
\hline \hline ACT-1 & $\begin{array}{l}\text { Sens-GACAATTTCTCTTTCAGCACTAGTAGTGA } \\
\text { Antisens-GCTGGTAGAGACTTGACCAACCA }\end{array}$ & 87 \\
\hline SAP2 & $\begin{array}{l}\text { Sens-TCCTGATGTTAATGTTGATTGTCAAG } \\
\text { Antisens-TGGATCATATGTCCCCTTTTGTT }\end{array}$ & 81 \\
\hline SAP4 & $\begin{array}{l}\text { Sens-AGATATTGAGCCCACAGAAATTCC } \\
\text { Antisens-CAATTTAACTGCAACAGGTCCTCTT }\end{array}$ & 81 \\
\hline SAP5 & $\begin{array}{l}\text { Sens-CATTGTGCAAAGTAACTGCAACAG } \\
\text { Antisens-CAGAATTTCCCGTCGATGAGA }\end{array}$ & 77 \\
\hline SAP6 & $\begin{array}{l}\text { Sens-CCTTTATGAGCACTAGTAGACCAAACG } \\
\text { Antisens-TTACGCAAAAGGTAACTTGTATCAAGA }\end{array}$ & 101 \\
\hline
\end{tabular}


(prepared as follows) were plated in duplicate and incubated for $1 \mathrm{~h}$ at $37^{\circ} \mathrm{C}$. The incubation mixtures, prepared in TBST $2 \% \mathrm{BSA}$, consisted of serial dilutions of rSap2, starting at $500 \mu \mathrm{g} / \mathrm{ml}$ (to obtain a Sap2 standard curve), or samples to be titrated, which were incubated in polystyrene tubes for $1 \mathrm{~h}$ at room temperature with an equal volume of anti-Sap2 IgG (diluted 1:75 in TBST 2\% BSA). After washing, the bound antibodies were detected by adding alkaline phosphatasecoupled monoclonal goat anti-mouse IgG (Southern Biotechnology Associates) for $30 \mathrm{~min}$ at room temperature. Substrate solution containing p-nitrophenyl phosphate (Sigma) was then added after washing and the reaction was stopped with the addition of $0.1 \mathrm{M}$ EDTA $\mathrm{pH}$ 8.0. Absorbance at $405 \mathrm{~nm}$ was inversely proportional to the amount of Sap present, and inhibition results were converted into $\mu \mathrm{g} / \mathrm{ml}$ of Sap by means of the obtained standard curve, which was linear over the concentration range used.

\section{Statistical Analyses}

Each experiment in this study was performed three times or more. Experimental values are given as means $+\mathrm{SD}$. The statistical significance of differences between the control values and the test values was evaluated using a one-way ANOVA. Posteriori comparisons were done using Tukey's method. Normality and variance assumptions were verified using the Shapiro-Wilk test and the Brown and Forsythe test, respectively. All of the assumptions were fulfilled. Data were analyzed using the SAS version 8.2 statistical package (SAS Institute Inc., Cary, NC, USA). Results were considered significant at $\mathrm{p}<0.05$.

\section{RESULTS}

\section{Inhibition of Germ Tube Formation by Farnesol}

As shown in Fig. (1), germ tube formation was reduced in the farnesol-treated $C$. albicans cells compared to that observed in the untreated control cultures Fig. (1A). The inhibition of germ tube formation was significant at both low and high concentrations of farnesol Fig. (1B) and as early as $6 \mathrm{~h}$ after contact. The inhibitory effect of farnesol on the yeast-to-hyphae transition was maintained after $24 \mathrm{~h}$ Figs. (1A and 1B). Only few, if any germ tubes were observed in the absence of BSA, either with or without farnesol (data not shown). The effect of farnesol against $C$. albicans transition could be through ultrastructural changes.

\section{Effect of Farnesol on C. Albicans Ultrastructure Changes}

The aerobic growth of $C$. albicans cells showed typical yeast cells displaying the characteristic bud scars (Fig. (2), SEM, Ctrl). No development of pseudohyphae (chains of elongated unseparated blastospores) was observed in the cultures with or without farnesol or Amph-B. Following the addition of farnesol, the external morphology of the cells did not appear as smooth as that of the untreated cells, which indicates a possible loss of cytosolic volume. Indeed, farnesol basically distorted the cell wall surface at high concentrations (Fig. (2), SEM). Nevertheless, the cell wall of $C$. albicans cultured in the presence of farnesol did not resemble to that of $C$. albicans treated with Amph-B. To confirm these results we performed transmission electron microscopy analyses. Incubation of $C$. albicans in the presence of farnesol

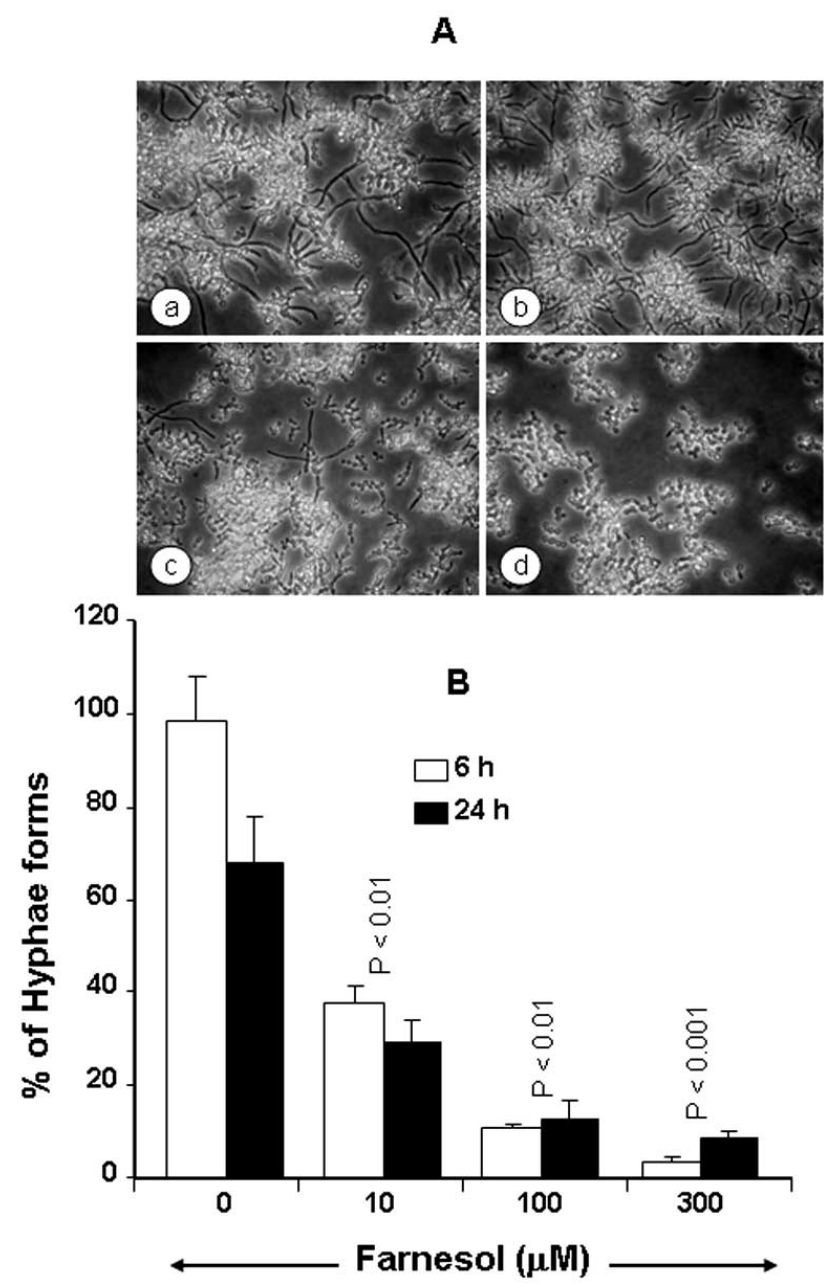

Fig. (1). Farnesol modulated Candida transition from blastospore to hyphae forms. C. albicans was cultured PIM containing or not BSA with and without farnesol at various concentrations (10, 100 , and $300 \mu \mathrm{M}$ ) for 6 and $24 \mathrm{~h}$ at $37^{\circ} \mathrm{C}$. After each time point, the cultures were observed under an inverted microscope and photographed. Panel A showed morphological changes at $6 \mathrm{~h}$. The numbers of yeast and hyphal forms were then counted. The percentage of hyphae was obtained by dividing the number of hyphae by the total number of cells (blastospores and hyphae) in each culture. The means + SD relative values are shown in (B). The levels of significance were obtained by comparing the percentages of yeast-tohyphae transition in the presence/absence of farnesol. (a), untreated C. albicans; (b), treated with $10 \mathrm{mM}$; (c), treated with $100 \mathrm{mM}$; (d), treated with $300 \mathrm{mM}$ of farnesol.

resulted in notable alterations in the internal morphology, when compared to control cells (Fig. (2), TEM). Non-treated C. albicans cells displayed a normal cellular morphology with typical dense cytoplasm and a distinct cell wall. Furthermore, untreated Candida showed continuous cytoplasmic membrane $(\mathrm{cm})$ lining a homogeneous and electron-dense cytoplasm that contains visible nucleus. However, in the presence of farnesol, large and irregular cytoplasmic vacuoles were detected. Peripheral vacuoles present in the farnesol-treated cells were not visible in organisms from the control cultures. Treatment with farnesol also resulted in an in- 


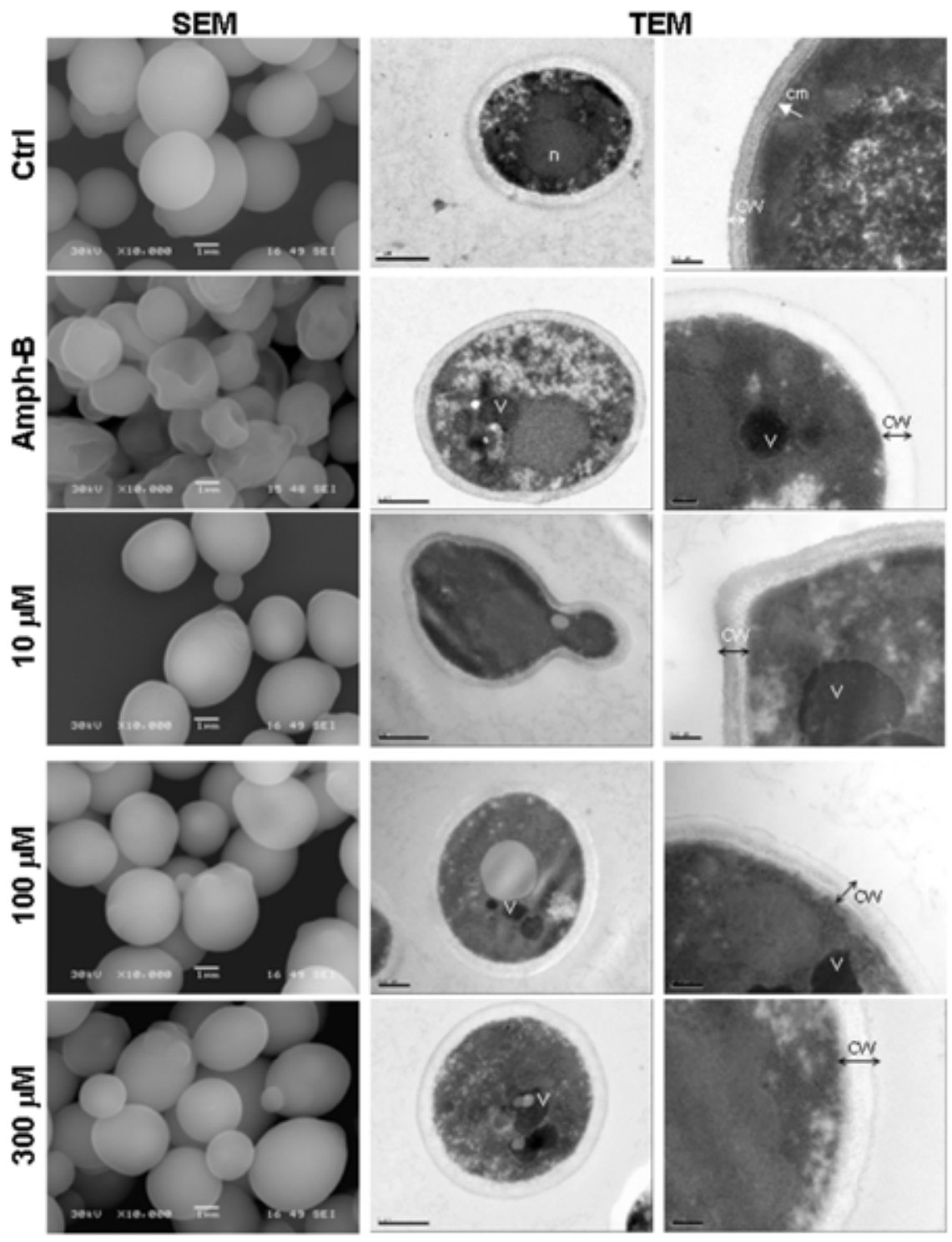

Fig. (2). Scanning electron microscopy (left column) and transmission electron microscopy (two right columns) micrographs of $C$. albicans with and without farnesol. Candida was untreated (Ctrl), treated with Ampho-B (positive control) or with farnesol at various concentrations for $24 \mathrm{~h}$ then subjected to scanning electron microscopy (SEM) and transmission electron microscopy (TEM) analyses. (n), nucleus; $(\mathrm{cm})$, continuous cytoplasmic membrane; (CW), Cell wall; (V), vacuole. Each experiment was repeated three and four times, for SEM and TEM, respectively.

creased granulation of the cytoplasm, diminished definition of the cell membrane as well as a loss of cell-wall integrity (Fig. (2), TEM). A similar effect was obtained when C. albicans was treated with Amph-B.

\section{Farnesol Modulated Sap2, 4, 5 and 6 mRNA Expressions}

As shown in Fig. (3), farnesol significantly $(p<0.01)$ affected Sap2 expression, showing decreased Sap2 mRNA expression at all of the tested concentrations. The decrease varied between 4- and 6-fold, compared to non-treated C. albicans cultures Fig. (3A). The same observations were made with Saps4-6. Farnesol significantly $(\mathrm{p}<0.05)$ downregulated mRNA expression of Saps4-6. The important effects were obtained with Sap5 and Sap6. With Sap5, the decrease varied between 2 and 5 folds Fig. (3C). With Sap6, the decrease was about 10 folds following $C$. albicans treat- ment with 100 and $300 \mu \mathrm{M}$ of farnesol Fig. (3D). With 10 $\mu \mathrm{M}$ of farnesol no effect was observed basically for Sap4 and Sap6.

\section{Farnesol Downregulated Sap2 Protein Production}

Whenever possible, mRNA expression should be confirmed by using protein expression analysis. As farnesol clearly downregulated Sap2 expression, and taking into account that Sap2 is the predominant Sap gene produced in vitro under most proteinase-inducing conditions, the effect of this quorum-sensing molecule on Sap2 protein production was thus investigated. To do so, anti-Sap2 IgG antibodies were produced to determine, by means of competitive binding inhibition ELISA, the concentration of Sap2 produced by C. albicans following treatment with farnesol. As shown in Fig. (4), Sap2 production was significantly reduced in the 

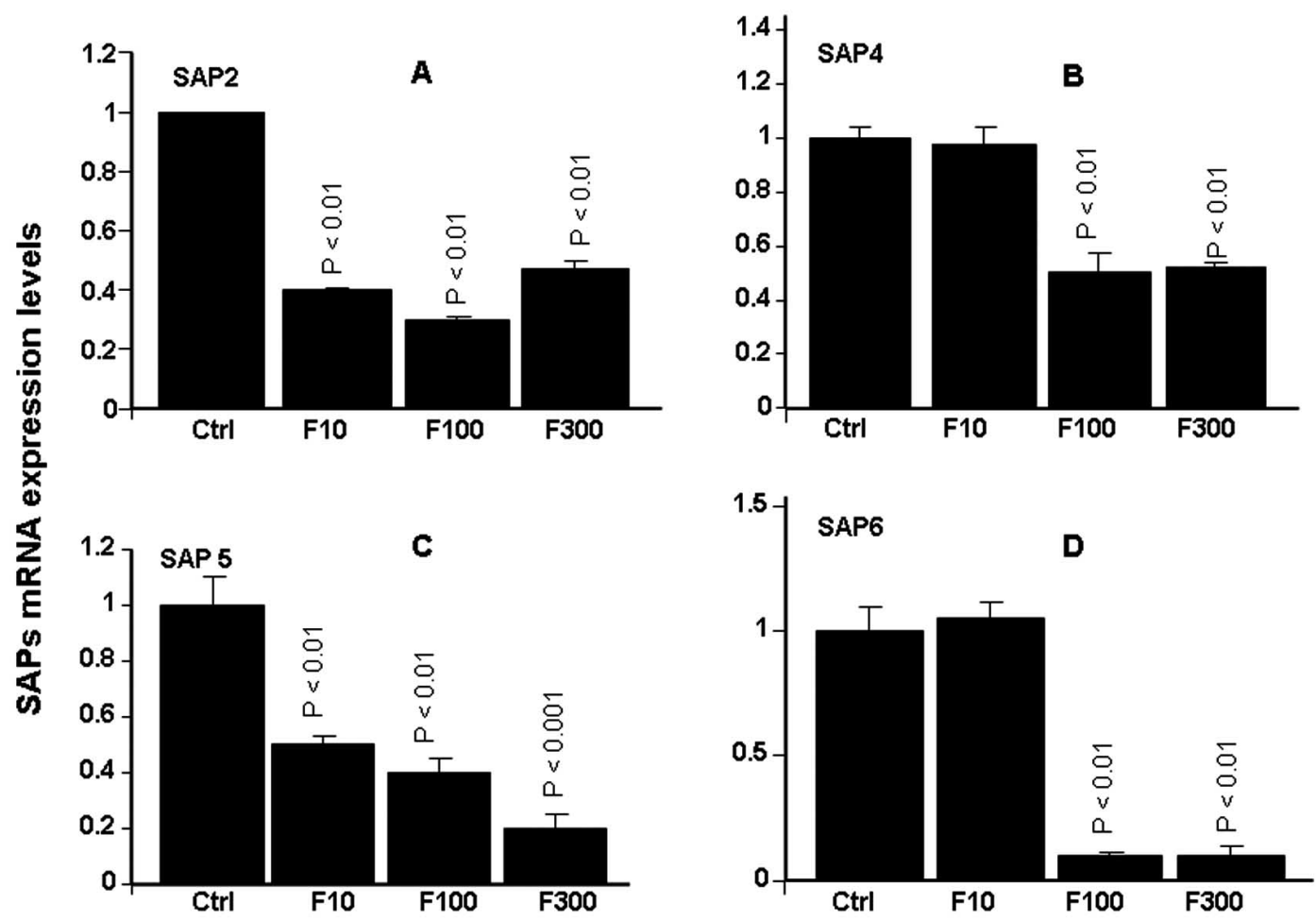

Fig. (3). Effect of farnesol on Sap2, 4, 5 and 6 genes expression. Following C. albicans culture in the presence of various concentrations of farnesol, total RNA was extracted from each cell culture and qRT-PCR was performed using specific primers for Sap2, 4, 5 and 6. ACT-1 was used as the housekeeping gene for internal control. The changes in mRNA levels are presented as the fold expression of the gene in the test sample compared to this gene's expression in the control (without farnesol). Data are expressed as means + SD from triplicate assays of three different experiments.

presence of farnesol. This observed reduction ranged between 4- and 16-fold and was dose-dependent. These results are in agreement with those of the Sap2 mRNA levels following farnesol treatment Fig. (3).

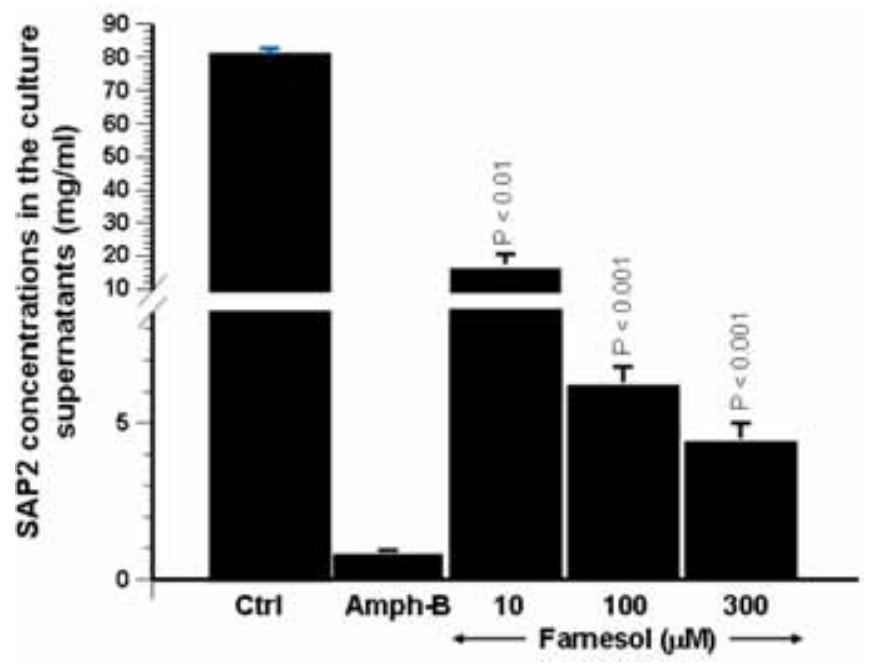

Fig. (4). Effect of farnesol on the secretion of Sap2 protein by $C$. albicans. Following Candida culture in the presence of various concentrations of farnesol for $24 \mathrm{~h}$, the supernatants were collected and used to quantify Sap2 level by competitive binding inhibition ELISA. The bars represent the means + SD of Sap2 concentration on three separate experiments. The levels of significance were obtained by comparing the values of farnesol stimulated cultures with control cultures.

\section{DISCUSSION}

Candida virulence is not limited only to growth, but is also mediated by its ability to change the morphotype from yeast to hyphae. In an experimental protocol that induce hyphae formation through the presence of BSA in the culture medium, we showed that farnesol reduced, up to 10 -fold, the Candida transition from yeast to hyphal forms. These data are in accordance with previously reported studies [36] showing that farnesol (which acts in an autocrine manner on Candida) blocks the morphological shift of Candida from yeast to hyphae at high cell densities $[37,38]$. This was confirmed by in vitro studies showing that exogenously-added farnesol inhibited germ tube formation [39-41] normally triggered by serum [32], proline and $\mathrm{N}$-acetylglucosamine [24]. Studies have shown that farnesol produced in situ by planktonic C. albicans cultures prevented biofilm formation [30]. To these we should add the present study showing for the first time that, exogenous farnesol modulates the behaviour of Candida that does not produce endogenous farnesol. Farnesol may therefore be a key player in modulating C. albicans pathogenesis. However, in a recent study, Weber et al. (2010) state that, farnesol may be involved in the virulence aspect of destroying the epithelial cell layer of the host as the initial invasion process [36]. Thus additional investigations that include Candida, exogenous farnesol and human epithelial cells are needed.

Farnesol is an amphiphilic molecule known to solubilise model membranes [42, 43]. In mammalian cells, it affects membrane ion channels [44] and in Staphylococcus aureus, 
it inhibits biofilm formation and compromises cell membrane integrity [45]. We therefore suggest that farnesol may modulate Candida growth by compromising the cell wall and/or cell membrane as well as cytoplasm integrity.

In this context, we performed ultrastructural analyses to show that farnesol distorted the cell wall surface at high concentrations. Farnesol-treated C. albicans displayed highly vacuolated cells with an increased granulation of the cytoplasm. Similar alterations were observed upon treatment of C. albicans cells with Ciclopirox [46], Amph-B, and nisin Z [47]. However, the mechanism involved in Candida growth control by farnesol remains to be elucidated. This may be linked to Candida interacting with its environment that involves $\mathrm{pH}$, temperature, and most certainly, the host. Compromising $C$. albicans cell wall and morphological changes from blastospore to hyphae could be through Saps genes modulation. It is well known that Candida pathogenesis may be reached through Candida growth as well as through its yeast-to-hyphae morphohenesis. Specific Saps were found to be preferentially expressed by Candida hyphae forms [20, 48]. The reduction of Sap expression by $C$. albicans may in fact reduce the yeast virulence/pathogenesis. We demonstrated that farnesol decreased Sap2 mRNA expression and protein production supporting the role of Sap2 in Candida in fulfilling basic functions in relation to transition and pathogenesis via proteinase [49], and the effect of farnesol on reducing Candida pathogenesis through Sap2 expression. These data are confirmed by some other Saps modulation. Indeed, Sap4-6 mRNA expressions were also downregulated upon farnesol treatment. This highlights, for the first time, a clear link between farnesol, hyphae morphogenesis, and hyphae-modulating genes (Sap4-6). Our findings support the usefulness of farnesol as a potential molecule to control Candida pathogenesis through Sap4-6 gene inhibiting. These Saps were previously reported to be involved in controlling Candida transition and virulence [48]. The precise interactions between the different Saps and farnesol during Candida pathogenesis remain unexplored. Further studies will focus on identifying the role of Sap subfamilies in Candida invasion, and the role of farnesol in controlling Candida virulence/pathogenesis in conjunction with host defenses.

In conclusion, this study demonstrates for the first time that exogenous farnesol used with $C$. albicans that does not endogenously produce farnesol downregulate hyphae morphogenesis through cell wall changes and Sap2, Saps4-6 mRNA expression decrease. Overall results suggest the possible use of farnesol as an antifungal molecule.

\section{ACKNOWLEDGEMENTS}

This study was supported by grants from the Natural Sciences and Engineering Research Council of Canada (NSERC) Discovery Program (MR).

\section{AUTHOR'S CONTRIBUTIONS}

Conceived and designed the experiments: MR and ND. Performed the experiments: ND, MR, MV and AC. Analyzed the data: ND, MR, AC and MV. MR and MV contributed reagents/ materials/ analysis tools. All authors wrote, read and approved the final manuscript.

\section{CONFLICTS OF INTEREST}

The authors have no conflicts of interest to report.

\section{REFERENCES}

[1] Wisplinghoff $\mathrm{H}$, Bischoff T, Tallent SM, Seifert H, Wenzel RP, Edmond MB. Nosocomial bloodstream infections in US hospitals: analysis of 24,179 cases from a prospective nationwide surveillance study. Clin Infect Dis 2004; 39: 309-17.

[2] Marchetti O, Bille J, Fluckiger U, et al. Epidemiology of candidemia in Swiss tertiary care hospitals: secular trends 1991-2000. Clin Infect Dis 2004; 38: 311-20.

[3] Cruciani M, de Lalla F, Mengoli C. Prophylaxis of Candida infections in adult trauma and surgical intensive care patients: a systematic review and metaanalysis. Intensive Care Med 2005; 31: 147987.

[4] Wey SB, Mori M, Pfaller MA, Woolson RF, Wenzel RP. Risk factors for hospital-acquired candidemia: a matched case-control study. Arch Intern Med 1989; 149: 2349-53.

[5] Menzin J, Meyers JL, Friedman M, et al. Mortality, length of hospitalization and costs associated with invasive fungal infections in high-risk patients. Am J Health Syst Pharm 2009; 66: 1711-7.

[6] Shorr AF, Gupta V, Sun X, Johannes RS, Spalding J, Tabak YP Burden of early-onset candidemia: analysis of culture-positive bloodstream infections from a large US database. Crit Care Med 2009; 37: 2519-26.

[7] Odds FC. Candida infections: an overview. Crit Rev Microbiol 1987; 15: 1-5.

[8] Sudbery P, Gow N, Berman J. The distinct morphogenic states of Candida albicans. Trends Microbiol 2004; 12: 317-24.

[9] Deslauriers N, Coulombe C, Carre B, Goulet JP. Topical application of a corticosteroid destabilizes the host-parasite relationship in an experimental model of the oral carrier state of Candida albicans. FEMS Immunol Med Microbiol 1995; 11: 45-55.

[10] Scully C, El-Kabir M, Samaranayake LP. Candida and oral candidosis: a review. Crit Rev Oral Biol Med 1994; 5: 125-57.

[11] Frohner IE, Bourgeois C, Yatsyk K, Majer O, Kuchler K. Candida albicans cell surface superoxide dismutases degrade host-derived reactive oxygen species to escape innate immune surveillance. Mol Microbiol 2009; 71: 240-52.

[12] Carlisle PL, Banerjee M, Lazzell A, Monteagudo C, López-Ribot JL, Kadosh D. Expression levels of a filament-specific transcriptional regulator are sufficient to determine Candida albicans morphology and virulence. Proc Natl Acad Sci USA 2009; 106: 599604.

[13] Tronchin G, Pihet M, Lopes-Bezerra LM, Bouchara JP. Adherence mechanisms in human pathogenic fungi. Med Mycol 2008; 46: 749-72.

[14] Phan QT, Myers C, Fu Y, Sheppard DC, et al. Als3 is a Candida albicans invasin that binds to cadherins and induces endocytosis by host cells. PLoS Biol 2007; 5: 543-57.

[15] Schaller M, Borelli C, Korting HC, Hube B. Hydrolytic enzymes as virulence factors of Candida albicans. Mycoses 2005; 48: 365-77.

[16] Naglik J, Albrecht A, Bader O, Hube B. Candida albicans proteinases and host/pathogen interactions. Cell Microbiol 2004; 6: 915 26.

[17] Naglik JR. Challacombe SJ, Hube B. Candida albicans secreted aspartyl proteinases in virulence and pathogenesis. Microbiol Mol Biol Rev 2003; 67: 400-28.

[18] Hube B, Naglik J. Candida albicans proteinases: resolving the mystery of a gene family. Microbiology 2001; 147: 1997-2005.

[19] White TC, Miyasaki SH, Agabian N. Three distinct secreted aspartyl proteinases in Candida albicans. J Bacteriol 1993; 175: 612633.

[20] White TC, Agabian N. Candida albicans secreted aspartyl proteinases: isoenzyme pattern is determined by cell type, and levels are determined by environmental factors. J Bacteriol 1995; 177: 5215-21.

[21] Albrecht A, Felk A, Pichova I, et al. Glycosylphosphatidylinositolanchored proteases of Candida albicans target proteins necessary for both cellular processes and host-pathogen interactions. J Biol Chem 2006; 281: 688-94.

[22] Odds F. Mycology in oral pathology. Acta Stomatol Belg 1997; 94 75-80. 
[23] Nickerson KW, Atkin AL, Hornby JM. Quorum sensing in dimorphic fungi: farnesol and beyond. Appl Environ Microbiol 2006; 72: 3805-13.

[24] Hornby JM, Jonsen EC, Lisec AD, et al. Quorum sensing in the dimorphic fungus Candida albicans is mediated by farnesol. Appl Environ Microbiol 2001; 67: 2982-92.

[25] Weber K, Sohr R, Schulz B, Fleischhacker M, Ruhnke M. Secretion of E,E-farnesol and biofilm formation in eight different Candida species. Antimicrob Agents Chemother 2008; 52: 1859-61.

[26] Ramage G, Saville SP, Wickes BL, Lopez-Ribot JL. Inhibition of Candida albicans biofilm formation by farnesol, a quorum-sensing molecule. Appl Environ Microbiol 2002; 68: 5459-63.

[27] Jensen EC, Hornby JM, Pagliaccetti NE, Wolter CM, Nickerson $\mathrm{KW}$, Atkin AL. Farnesol restores wild-type colony morphology to 96\% of Candida albicans colony morphology variants recovered following treatment with mutagens. Genome 2006; 49: 346-53.

[28] Braun PC. The effect of farnesol on amino acid incorporation by a wild-type and cell-wall variant strain of Candida albicans. Can J Microbiol 2005; 51: 715-8

[29] Enjalbert B, Whiteway M. Release from quorum-sensing molecules triggers hyphal formation during Candida albicans resumption of growth. Eukaryot Cell 2005; 4: 1203-10.

[30] Hornby JM, Nickerson KW. Enhanced production of farnesol by Candida albicans treated with four azoles. Antimicrob Agents Chemother 2004; 48: 2305-7.

[31] Falkensammer B, Pleyer L, Ressler S, et al. Basidiomycete metabolites attenuate virulence properties of Candida albicans in vitro. Mycoses 2008; 51: 505-14.

[32] Saidi S, Luitaud C, Rouabhia M. In vitro synergistic effect of farnesol and human gingival cells against Candida albicans. Yeast 2006; 23: 673-87.

[33] Ausubel F M, Brent B, Kingston RE, et al. editors Current Protocols In Molecular Biology. New York, USA: John Wiley \& Sons Inc.; 1994.

[34] Naglik JR, Moyes D, Makwana J, et al. Quantitative expression of the Candida albicans secreted aspartyl proteinase gene family in human oral and vaginal candidiasis. Microbiology 2008; 154: 3266-80.

[35] Vilanova M, Teixeira L, Caramalho I, et al. Protection against systemic candidiasis in mice immunized with secreted aspartic proteinase 2. Immunology 2004; 111: 334-42.

[36] Mosel DD, Dumitru R, Hornby JM, Atkin AL, Nickerson KW. Farnesol concentrations required to block germ tube formation in
Candida albicans in the presence and absence of serum. Appl Environ Microbiol 2005; 71: 4938-40.

[37] Weber K, Schulz B, Ruhnke M. The quorum-sensing molecule E,E-farnesol--its variable secretion and its impact on the growth and metabolism of Candida species. Yeast 2010; 27: 727-39.

[38] Joo JH, Jetten AM. Molecular mechanisms involved in farnesolinduced apoptosis. Cancer Lett. 2010; 287: 123-35.

[39] Uppuluri P, Mekala S, Chaffin WL. Farnesol-mediated inhibition of Candida albicans yeast growth and rescue by a diacylglycerol analogue. Yeast 2007; 24: 681-93

[40] Davis-Hanna A, Piispanen AE, Stateva LI, Hogan DA. Farnesol and dodecanol effects on the Candida albicans Ras1-cAMP signalling pathway and the regulation of morphogenesis. Mol Microbiol 2008; 67: 47-62.

[41] Shirtliff ME, Krom BP, Meijering RA, et al. Farnesol-induced apoptosis in Candida albicans. Antimicrob Agents Chemother 2009; 53: 2392-401.

[42] Funari SS, Prades J, Escribá PV, Barceló F. Farnesol and geranylgeraniol modulate the structural properties of phosphatidylethanolamine model membranes. Mol Membr Biol 2005; 22: 303-11.

[43] Rowat AC, Keller D, Ipsen JH. Effects of farnesol on the physical properties of DMPC membranes. Biochim Biophys Acta 2004; 1713: 29-39.

[44] Bringmann A, Skatchkov S, Faude F, Enzmann V, Reichenbach A. Farnesol modulates membrane currents in human retinal glial cells. J Neurosci Res 2000, 62: 396-402.

[45] Jabra-Rizk MA, Meiller T, James CE, Shirtliff ME. Effect of farnesol on Staphylococcus aureus biofilm formation and antimicrobial susceptibility. Antimicrob Agents Chemother 2006; 50: 1463-9.

[46] Niewerth M, Kunze D, Seibold M, Schaller M, Korting HC, Hube B. Ciclopirox olamine treatment affects the expression pattern of Candida albicans genes encoding virulence factors, iron metabolism proteins, and drug resistance factors. Antimicrob Agents Chemother 2003; 47: 1807-17.

[47] Le Lay C, Akerey B, Fliss I, Subirade M, Rouabhia M. Nisin Z inhibits the growth of Candida albicans and its transition from blastospore to hyphal form. J Appl Microbiol 2008; 105: 1630-9.

[48] Hube B, Monod M, Schofield DA, Brown AJ, Gow NA. Expression of seven members of the gene family encoding secretory aspartyl proteinases in Candida albicans. Mol Microbiol 1994; 14: 87-99.

[49] Lian CH, Liu WD. Differential expression of Candida albicans secreted aspartyl proteinase in human vulvovaginal candidiasis. Mycoses 2007; 50: 383-90. 